\title{
New name, new team, new system
}

\author{
PAUL GRANT
}

The keen-eyed among you may have spotted something slightly different about the front cover this quarter. We have decided to 'drop the VD' from the title of the ABCD society journal. This is not because we are no longer interested in vascular disease - on the contrary, this clearly remains a key element of what is important to us as diabetologists. Merely, the name shortening reflects a further step in the evolution of the journal, a clear focus of what we are about and the directness of our message and content.

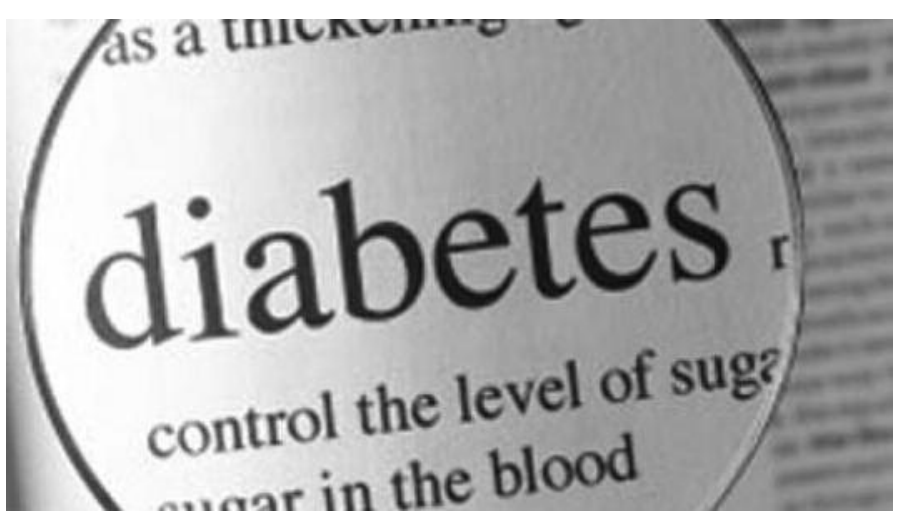

Under the bonnet there are other refinements, including the addition of several new associate editors to help with the running of the journal and copyflow (including Dr. Hermione Price, Dr. Roselle
Herring, Dr. Marie-France Kong, Dr. Dipesh Patel and Dr. Parth Narendran). Soon we will appoint a new editorial registrar (a diabetologist in training) and a new online manuscript handling system, which will help with the tracking of papers and aid with the turnaround time of peer review and editing - we are moving out of the clunky phase of back and forth email attachments and into something much more 21st century.

This issue includes multiple articles from the broad church that constitutes diabetes care, from an editorial on the work of DUK from its new CEO, to comment on the junior doctor's debacle from the YDEF and original reviews, research and audit work, including a good look at the efficacy of concentrated insulins and the latest in our Dermatology and Diabetes series. Finally a reminder from Andy Macklin to ensure that you keep up to date with the latest news from $A B C D$ by following on @ABCDiab.

If you have something you wish to share with the wider diabetes professional community, then find the online journal submissions system coming soon at http://www.bjd-abcd.com.

Editor-in-Chief, BJD

Address for correspondence: Paul Grant E-mail: editor@bjd-abcd.com

Br J Diabetes 2016;16:1

http://dx.doi.org/10.15277/bjd.2016.069

\section{$\overrightarrow{A B C D}$ \\ Insulin degludec (Tresiba) Nationwide Audit in progress}

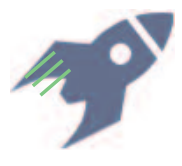

$A B C D$ has launched a nationwide audit of insulin degludec in the UK to assess real clinical efficacy and safety \& inform future practice and guidelines

\section{Does your centre use insulin degludec?}

If yes, REGISTER YOUR CENTRE! by contacting degludec.audit@diabetologists.org.uk

- you are invited to enter your patients' data into the bespoke online tool

- you are able to analyse your local data easily

- the data will be automatically added to the national data in anonymised form

- we can provide easy-to-complete paper proformas for use in clinic if preferred

Please remember: - the more data, the more complete our understanding of insulin degludec in real clinical practice will be

- all contributors will be listed in publications arising from data submission 